\title{
Comparison of the incidence, clinical features and outcomes of invasive candidiasis in children and neonates
}

\author{
Jen-Fu Hsu ${ }^{1,3}$, Mei-Yin Lai ${ }^{1,3}$, Chiang-Wen Lee ${ }^{4}$, Shih-Ming Chu ${ }^{1,3}$, I-Hsyuan Wu ${ }^{1,3}$, Hsuan-Rong Huang ${ }^{1,3}$, I-Ta Lee ${ }^{5}$,
} Ming-Chou Chiang ${ }^{1,3}$, Ren-Huei Fu ${ }^{1,3}$ and Ming-Horng Tsai ${ }^{2,3^{*}}$

\begin{abstract}
Background: Invasive candidiasis differs greatly between children and neonates. We aimed to investigate the different therapeutic approaches and their effects on treatment outcomes of these two groups.

Methods: Episodes of neonatal invasive candidiasis were compared with non-neonatal pediatric episodes during a 12-year cohort study. Clinical isolates were documented by matrix-assisted laser desorption/ionization-time of flight mass spectrometry and DNA sequencing, and antifungal susceptibility testing was performed.

Results: A total of 342 episodes of invasive candidiasis (113 neonatal and 229 non-neonatal pediatric episodes) in 281 pediatric patients (96 neonates and 185 children) were identified. Candida albicans was the most common pathogen causing invasive candidiasis in neonates and children (47.8\% vs. $44.1 \%)$. The antifungal susceptibility profiles were not significantly different between neonates and children. More neonates received amphotericin B as therapy, whereas more children received fluconazole or caspofungin. Compared with children, neonates had a significantly longer duration of fungemia, higher rates of septic shock (34.5\% vs. $21.8 \%$; $P=0.013)$, sepsis-attributable mortality $(28.3 \%$ vs. $17.5 \% ; P=0.024)$ and in-hospital mortality $(42.7 \%$ vs. $25.4 \% ; P=0.004)$ than children. Independent risk factors for treatment failure of invasive candidiasis were septic shock (odds ration [OR] 16.01; 95\% confidence interval [Cl] 7.64-33.56; $P<0.001$ ), delayed removal of intravenous catheter (OR 6.78; 95\% Cl 2.80-17.41; $P<0.001$ ), renal failure (OR 5.38; 95\% Cl 1.99-14.57; $P=0.001)$, and breakthrough invasive candidiasis (OR 2.99; 95\% Cl 1.04-8.67; $P=0.043)$.
\end{abstract}

Conclusions: Neonatal invasive candidiasis has worse outcomes than non-neonatal pediatric candidiasis. Neonatologists and pediatricians must consider age-specific differences when developing treatment and prevention guidelines, or when interpreting studies of other age groups.

Keywords: Bloodstream infection, Invasive candidiasis, Candidemia, Antifungal susceptibility, Mortality

\section{Background}

Candida species are the fourth most common cause of nosocomial infection and are the leading cause of invasive fungal infection among hospitalized patients $[1,2]$. Invasive candidiasis deserves greater attention because it is associated with a high mortality rate, especially in severely ill patients [3-5]. Recent population-based

\footnotetext{
* Correspondence: mingmin.tw@yahoo.com.tw

${ }^{2}$ Division of Neonatology and Pediatric Hematology/Oncology, Department

of Pediatrics, Chang Gung Memorial Hospital, No.707, Gongye Rd., Sansheng,

Mailiao Township, Yunlin, Taiwan, Republic of China

${ }^{3}$ College of Medicine, Chang Gung University, Taoyuan, Taiwan

Full list of author information is available at the end of the article
}

surveillance studies have shown an increased incidence of invasive candidiasis in intensive care units (ICUs) during the past decade $[6,7]$. An increase of susceptible hosts who receive intensive care or immunosuppressive therapies and the widespread use of broad-spectrum antibiotics may account for the increase of invasive Candida infections [8-10]. Furthermore, uses of antifungal drugs such as azoles for prophylaxis and echinocandins for treatment are reported to be associated with a continuous shift from $C$. albicans to various non-albicans Candida species [11, 12].

(c) The Author(s). 2018 Open Access This article is distributed under the terms of the Creative Commons Attribution 4.0 International License (http://creativecommons.org/licenses/by/4.0/), which permits unrestricted use, distribution, and reproduction in any medium, provided you give appropriate credit to the original author(s) and the source, provide a link to the Creative Commons license, and indicate if changes were made. The Creative Commons Public Domain Dedication waiver (http://creativecommons.org/publicdomain/zero/1.0/) applies to the data made available in this article, unless otherwise stated. 
The microbiological and clinical characteristics of invasive Candida infections vary widely among different geographic areas, patient characteristics and ages, and institutions [13, 14]. Although some studies concluded that the mortality of candidemia was higher in adults than in children [15], a recent study found a poor prognosis among infants $(<1$ year of age) and elderly patients (>60 years) [16]. Furthermore, we recently documented fungemia as an independent risk factor for treatment failure in the neonatal ICU (NICU) [17]. The reported incidence of candidemia in pediatric patients generally ranges between 0.21 and 10.5 cases per 1000 admissions $[15,18-20]$; however, patients in the NICU, pediatric ICU (PICU), and pediatric wards were not studied as separate and distinct groups $[15,21]$. In order to clarify and assess unique characteristics of invasive candidiasis in neonates and children, we compared the epidemiology and clinical features of Candida spp. identified by matrix-assisated laser desorption/ionization time-offlight mass spectrometry (MALDI-TOF) causing invasive candidiasis in these two populations.

\section{Methods}

We included all hospitalized patients in the Department of Pediatrics, Chang Gung Memorial Hospital (CGMH) from January 2004 through December 2015, for whom $\geq 1$ blood culture and/or sterile site cultures were positive for Candida spp. and who had symptoms, signs, or laboratory findings consistent with fungal infection. We retrospectively reviewed electronic medical records for demographic, clinical and laboratory data for the onset of invasive candidiasis (defined as the day of blood or sterile site collection for culture), and we reviewed risk factors within the preceding 30 days, major comorbidities, complications of invasive candidiasis, treatments and outcomes. The study was approved by the Institutional Review Board and Human Research Ethics Committee of CGMH, and a waiver of informed consent for anonymous data collection was also approved.

Isolation and identification of all Candida spp. isolates in blood and sterile site cultures were performed using a standard API 32C AUX yeast identification kit (bioMérieux SA, Marcy l'Étoile, France) and chromogenic culture media (CHROMagar; Becton Dickinson and Company, USA). Since December 2013, we have used MALDI-TOF (Bruker Biotype, software version 3. 0, Ewing, NJ, USA) and large-subunit (18S) ribosomal RNA gene D1/D2 domain sequencing to re-confirm all these species. Antifungal susceptibility was tested using the Clinical and Laboratory Standards Institute broth microdilution reference method [22]. For uncommon Candida spp., clinical breakpoints are undefined; therefore, isolates that showed minimum inhibitory concentrations (MICs) higher than the epidemiologic cutoff value were considered potentially resistant [23]. We excluded unidentified Candida species and selected only the first isolate recovered from the blood or sterile sites if a patient had several cultures that were positive for the same Candida spp.

\section{Definitions}

Invasive candidiasis included candidemia and deepseated candidiasis, which were defined as the recovery of a Candida species from blood or a sterile site, respectively $[24,25]$. An episode of candidemia was considered to be catheter-related only if the catheter tip culture was positive for the same Candida spp. [26]. Episodes were considered to be separate if they occurred $\geq 1$ month apart. Breakthrough invasive fungal disease was defined as candidemia or positive Candida spp. isolated from a sterile site in a patient who had undergone therapy or prophylaxis with any systemic antifungal drug for $\geq 3$ consecutive days before the index blood culture $[8,27]$. Invasive candidiasis-attributable mortality was defined when the patient died within 7 days after onset of invasive candidiasis or in the presence of persistent clinical sepsis or persistent candidemia, or if the patient died of candidemia associated complications [27, 28]. Combined with the antifungal susceptibility results, treatment failure was defined as an infection that led to attributable mortality or cases of persistent candidemia $\geq 7$ days after initiation of effective antifungal therapy. Patient responses to antifungal therapy following invasive candidiasis were defined according to the consensus criteria of the Mycoses Study Group and the European Organization for Research and Treatment of Cancer [29].

\section{Statistical analysis}

Clinical data were analyzed using SPSS version 18.0 (SPSS Inc., Chicago, IL, USA). Variables associated with invasive candidiasis in the NICU were compared with non-neonatal episodes. Univariate analyses were performed using Student's $t$-test or non-parametric tests as appropriate (for continuous variables) or the chi-square or Fisher's exact tests (for categorical variables). All tests were 2 -tailed, and a $P$ value of $<0.05$ was considered significant. We performed multiple logistic regression analyses to identify clinical risk factors that were associated with treatment failure of invasive candidiasis. All risk factors that were significant at 0.10 in the univariate analysis were included in the corresponding multivariate analysis.

\section{Results}

Incidence and demographic data

Among a total of 20,545 neonatal admissions and 153,372 pediatric non-neonatal admissions (of which 14,018 were PICU admissions), there were a total of 342 
invasive candidiasis episodes in 281 children that occurred during the study period; of these, neonatal episodes accounted for 113 episodes (33.0\%, in 96 neonates). The incidence rates of invasive candidiasis in neonates (NICU) and non-neonatal pediatric patients were 26.9 episodes per 100,000 inpatient days and 32.6 episodes per 100,000 inpatient days, respectively. However, the incidence rate of invasive candidiasis in the PICU was 147.2 episodes per 100,000 inpatients days, which was significantly higher than the rates in the NICU and in the general pediatric ward (both $P<0$. 001). Overall, the annual incidence rates of invasive candidiasis did not change significantly throughout the study period and varied between 27.3 and 36.7 episodes per 100,000 inpatients days (data not shown).

Invasive candidiasis occurred in 152 boys $(54.1 \%$ of all patients) and 129 girls. The mean age for non-neonatal pediatric patients was $6.2 \pm 5.7$ years (range, 3 months to 18 years). Overall, 214 (62.6\%) episodes of invasive candidiasis occurred in children $\leq 3$ years old (Fig. 1). Most of the invasive candidiasis episodes were primary bloodstream infections (228 episodes, 66.7\%), followed by catheterrelated bloodstream infections (69 episodes, 20.2\%), and intra-abdominal infections (31 episodes, 9.1\%). The sites of isolation and Candida species distributions were not significantly different between the neonatal and nonneonatal groups (Table 1), except that only two episodes in the NICU were caused by $C$. tropicalis. C. albicans was the most common Candida species that caused invasive candidiasis in children (45.3\%, 155 episodes), followed by C. parapsilosis (27.8\%, 95 episodes), C. tropicalis (6.4\%, 22 episodes) and C. glabrata (6.1\%, 21 episodes). Polyfungal isolates (i.e., two different Candida species yielded on cultures of blood samples that were obtained simultaneously) were recovered from three episodes, and the majority of ascites cultures (in 23 episodes, 74.2\%) were polymicrobial isolates that also yielded gram-positive cocci, or aerobic and anaerobic gram-negative bacilli.

\section{Risk factors}

The majority of patients had multiple underlying illnesses and other risk factors that have been associated with invasive candidiasis (Table 2). The majority of neonatal invasive candidiasis cases occurred in very low birth weight infants (birth weight $<1500 \mathrm{~g}$ ) (98 episodes, $86.7 \%$ ), and the mean (SD) gestational age in this group was $27.8 \pm 3.9$ weeks. The most common predisposing factors were use of central intravenous catheter (CVC) (94.2\%), use of broad-spectrum antibiotics (91.8\%), stay in an ICU (69.3\%), receipt of parenteral nutrition (64. $6 \%$ ), and underlying neurological sequelae (36.0\%). For 282 episodes $(82.4 \%), \geq 4$ risk factors and/or underlying illness were identified. However, the underlying illness and major predisposing factors were significantly different between neonatal invasive candidiasis and nonneonatal pediatric episodes. While neonates with invasive candidiasis were significantly more likely to have chronic lung disease and receive total parenteral nutrition, non-neonatal pediatric candidemia were more likely to occur in children with underlying neurological sequelae, cancer treated immunosuppressive agents, neutropenia and artificial devices other than CVC.

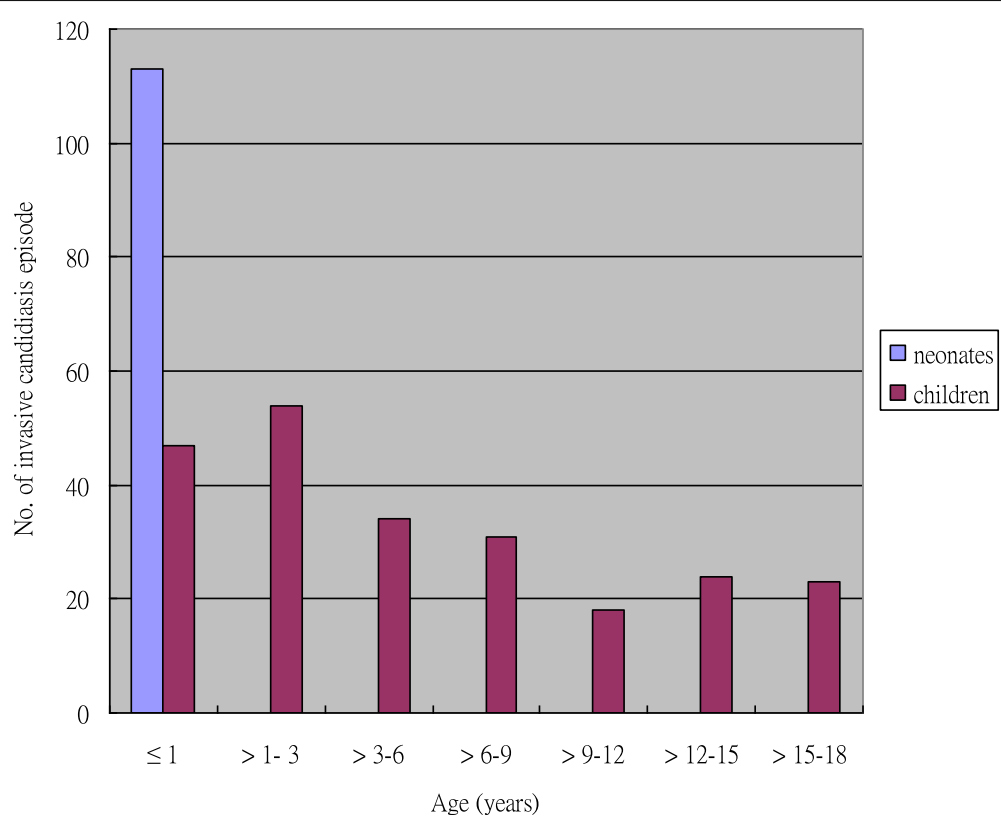

Fig. 1 The age distribution of the occurrence of invasive candidiasis in children 
Table 1 Incidence and Candida spp. causing invasive candidiasis over a 12-year period in children

\begin{tabular}{|c|c|c|c|}
\hline & \multirow{2}{*}{$\begin{array}{l}\text { Neonatal } \\
\text { episodes } \\
\text { (NICU) }\end{array}$} & \multicolumn{2}{|c|}{ Non-neonatal episodes } \\
\hline & & $\mathrm{PICU}$ & General wards \\
\hline Total episodes & $113(33.0)$ & $124(36.2)$ & $105(30.7)$ \\
\hline \multicolumn{4}{|l|}{ Incidence rate } \\
\hline per 100,000 inpatient days & 26.9 & 147.2 & 16.7 \\
\hline per 10,000 admissions & 55.0 & 88.5 & 7.5 \\
\hline \multicolumn{4}{|l|}{ Pathogens } \\
\hline Candida albicans & $54(47.8)$ & $61(49.2)$ & $40(38.1)$ \\
\hline Candida parapsilosis & $32(28.3)$ & $29(23.4)$ & $34(32.4)$ \\
\hline Candida tropicalis & $2(1.8)$ & $13(10.5)$ & $7(6.7)$ \\
\hline Candida glabrata & $10(8.8)$ & $7(5.6)$ & $4(3.8)$ \\
\hline Others & $15(13.3)$ & $14(11.3)$ & $20(19.0)$ \\
\hline \multicolumn{4}{|l|}{ Sites of isolations } \\
\hline Blood & 103 & 118 & 97 \\
\hline Central venous catheter $^{a}$ & 22 & 29 & 18 \\
\hline Abdomen & 9 & 12 & 6 \\
\hline Urine $^{b}$ & 4 & 7 & 2 \\
\hline Cerebrospinal fluid & 2 & 2 & 1 \\
\hline Lung $^{c}$ & 0 & 4 & 0 \\
\hline
\end{tabular}

NICU neonatal intensive care unit, PICU pediatric intensive care unit ancluding Port-A catheter, Hickman catheter, and percutaneous inserted central venous catheter

${ }^{\mathrm{b}}$ Suprapubic puncture for collection of urine in the NICU, and catheterization for collection of specimen in non-neonatal children

${ }^{c}$ Cultures from pleural fluid

\section{Clinical presentations}

No patient developed endophthalmitis, endocarditis, or osteomyelitis that was clinically evident, but five had CNS infection and four had an obstructing renal fungus ball during the follow-up period. In addition, 31 had intraabdominal abscesses or peritonitis and four patients had positive Candida isolates from pleural fluids. $36.8 \%$ of invasive candidiasis presented with severe sepsis, and $26.0 \%$ had septic shock at the onset of sepsis. After effective antifungal treatment, $17.8 \%$ had progressive and deteriorated candidiasis, and 14 (4.1\%) had disseminated candidiasis. Neonates with invasive candidiasis had significantly higher severity of illness than children based on the surrogate marker of severe sepsis and septic shock (Table 3).

More than half of the episodes (180 episodes, 52.6\%) were characterized by fungemia or persistent invasive candidiasis of > 1 day's duration, whereas 165 (48.2\%) and $78(22.8 \%)$ were characterized by fungemia or persistent invasive candidiasis of $\geq 3$ days and $\geq 7$ days, respectively. The mean duration of fungemia was 4.4 days ( \pm 6.2 days). The longest duration of fungemia was 34 days, which occurred in a patient who had end-stage renal disease and who received long term hemodialysis.

\section{Treatment and outcomes}

Of the 342 episodes, 332 (97.1\%) were treated with an antifungal agent, and there were 41 episodes of breakthrough invasive candidiasis. Ten episodes (2. 9\%) were not treated because of the patient's death before or at the time of the diagnosis was established. Antifungal therapy was initiated after a mean of 1 . 81 days (range, 0-6) following the acquisition of the first diagnostic blood and/or sterile site culture and was significantly later in neonates than in children (2. $1 \pm 1.3$ vs. $1.7 \pm 1.4$ days, $P=0.009$ ). The mean duration of all antifungal therapy per episode was 18 . 5 days (range, 1-68). Of those 332 episodes for which an antifungal agent was used, 151 episodes (45.5\%) had modification of the antifungal regimens during the treatment course, mainly due to the patient's poor response to initial antifungal therapy (101 episodes, $66.9 \%)$, suspicious antifungal resistance after confirmation of Candida spp.(36 episodes, $23.8 \%$ ), or no reason was documented (14 episodes, 9.3\%). Among the antifungal regimens for treatment, fluconazole was the most commonly prescribed initially (62.3\%), followed by amphotericin B (24.7\%) and caspofungin (4.5\%). However, the final treatment regimens were fluconazole/Voriconazole (39.5\%), amphotericin B (29.2\%) and echinocandin (28.9\%), with significant differences between neonates and children (Table 3 ).

Catheter removal was done within 3 days after illness onset in only one-third of patients with invasive candidiasis (107 episodes, 32.2\%), and in 22 episodes, the candidemia resolved only after removal of the intravenous catheter. Neonates with invasive candidiasis had a longer period of fungemia than children, and a higher rate of treatment failure was also noted $(31.0 \%$ vs. $19.7 \%, P=0$. 015) (Table 3). Invasive candidiasis in neonates was associated with a significantly higher rate of sepsisattributable mortality than that in children $(28.3 \%$ vs. $17.5 \%, P=0.024)$. After invasive candidiasis, neonates had a higher rate of in-hospital mortality than children $(42.7 \%$ vs. $25.4 \%, P=0.004$, and $P=0.005$ by $\log$ rank test [Fig. 2]).

\section{Susceptibility studies}

In vitro susceptibility to various commonly prescribed antifungal agents in our hospital was determined for 295 isolates (Table 4). The rate of fluconazole-R or SDD Candida was $14.6 \%$ (43 of 295 isolates) overall. The antifungal susceptibility profiles of Candida spp. in neonates were not significantly different between those in non-neonatal pediatric episodes. No trend toward higher minimum inhibitory concentrations was noted when earlier isolates (i.e., isolates recovered during 2004-2009) were compared with those obtained later (i.e., those recovered during 2010-2015). 
Table 2 Demographic and clinical characteristics of 342 episodes of invasive candidiasis in neonatal versus non-neonatal pediatric children

\begin{tabular}{|c|c|c|c|}
\hline Characteristic & $\begin{array}{l}\text { Neonatal episodes } \\
\text { (total } n=113 \text { ) }\end{array}$ & $\begin{array}{l}\text { Non-neonatal pediatric episodes } \\
\text { (total } n=229 \text { ) }\end{array}$ & $P$ value \\
\hline Patient age, median (IQR) & $27.0(19.0-56.0)$ days & $3.8(1.2-10.6)$ years & - \\
\hline Sex, male subjects/female subjects & $68(60.2) / 45(39.8)$ & $110(48.0) / 119(52.0)$ & 0.039 \\
\hline Gestational age (weeks), median (IQR) & $27.0(25.0-29.0)$ & - & - \\
\hline \multicolumn{4}{|l|}{ Underlying conditions $^{\mathrm{a}}$} \\
\hline Congenital or genetic anomalies & $9(8.0)$ & $25(10.9)$ & 0.447 \\
\hline Neurological sequelae & $22(19.5)$ & $101(44.1)$ & $<0.001$ \\
\hline Cardiovascular disease & $9(8.0)$ & $22(9.6)$ & 0.693 \\
\hline Chronic lung disease and/or pulmonary hypertension & $60(53.1)$ & $33(14.4)$ & $<0.001$ \\
\hline Gastrointestinal sequelae & $26(23.0)$ & $69(30.1)$ & 0.119 \\
\hline Renal sufficiency with/without dialysis & $8(7.1)$ & $31(13.5)$ & 0.103 \\
\hline Hematological/Oncology cancer & $0(0)$ & $48(21.0)$ & $<0.001$ \\
\hline Immunodeficiency & $1(0.9)$ & $6(2.6)$ & 0.344 \\
\hline Autoimmune disease & $0(0)$ & $8(3.5)$ & 0.056 \\
\hline Hepatic failure or cholestasis & $4(3.5)$ & $9(3.9)$ & 1.000 \\
\hline Others $^{b}$ & $1(0.9)$ & $1(0.4)$ & - \\
\hline $\begin{array}{l}\text { Days of hospitalization before onset of invasive candidiasis, } \\
\text { median (IQR) }\end{array}$ & $26.0(17.0-55.0)$ & $29.5(13.0-49.0)$ & 0.818 \\
\hline Sequences of episodes & & & 0.371 \\
\hline First episode & $96(85.0)$ & $185(80.8)$ & \\
\hline Recurrent episode & $17(15.0)$ & $44(19.2)$ & \\
\hline \multicolumn{4}{|l|}{ Associated risk factors ${ }^{b}$} \\
\hline Receipt of systemic antibiotics ${ }^{c}$ & $106(93.8)$ & $208(90.8)$ & 0.407 \\
\hline Prior bacteremia $^{c}$ & $43(38.1)$ & $126(55.0)$ & 0.004 \\
\hline Prior azoles exposure ${ }^{c}$ & $10(8.8)$ & $21(9.2)$ & 1.000 \\
\hline Presence of central venous catheter & $108(95.6)$ & $214(93.4)$ & 0.625 \\
\hline Stay in an intensive care unit & $113(100)$ & $124(54.1)$ & $<0.001$ \\
\hline Receipt of parenteral nutrition & $105(92.9)$ & $116(50.7)$ & $<0.001$ \\
\hline Receipt of immunosuppressive drugs & $3(2.7)$ & $65(28.4)$ & $<0.001$ \\
\hline Presence of artificial device other than central venous catheter & $34(30.1)$ & $133(58.1)$ & $<0.001$ \\
\hline Prior surgery ${ }^{c}$ & $31(27.4)$ & $79(34.5)$ & 0.219 \\
\hline Neutropenia $^{d}$ & $12(10.6)$ & $70(30.6)$ & $<0.001$ \\
\hline
\end{tabular}

All data were expressed as number (percentage \%), unless indicated otherwise; IQR interquartile range

andicated the presence of underlying condition or risk factor at onset of invasive candidiasis, and most episodes occurred in patients with > 1 underlying condition or risk factor

${ }^{\mathrm{b}}$ One neonatal episode occurred in a patient with epidermolysis bullosa, and one non-neonatal pediatric episode occurred in a patient with diabetes mellitus 'Within 1 month prior onset of invasive candidiasis

${ }^{\mathrm{d}}$ Absolute neutrophil count $\leq 500$ cells/ $\mu \mathrm{L}$

\section{Independent risk factors for treatment failure}

Except for underlying renal failure that required hemodialysis, none of underlying chronic comorbidities were associated with treatment failure. Treatment failure was not associated with any specific Candida species that caused invasive candidiasis. After multivariate logistic regression analysis (Table 5), the independent risk factors for treatment failure of invasive candidiasis included septic shock (odds ratio [OR]: 16.01; 95\% confidence interval [CI]: 7.64-33.56; $P$
$<0.001$ ), delayed removal of intravenous catheter (after 3 days of disease onset) (OR: 6.78; 95\% CI: 2.48-18.52; $P$ $<0.001$ ), underlying renal failure with/without hemodialysis (OR: 5.38; 95\% CI: 1.99-14.57; $P=0.001$ ), and breakthrough invasive candidiasis (OR: 2.99; 95\% CI: 1.04-8.67; $P=0.043$ ).

\section{Discussion}

The epidemiology and choice of therapy for candidemia or invasive candidiasis are rapidly changing, and vary 
Table 3 Clinical features, treatment and outcomes of invasive candidiasis in neonatal episodes versus non-neonatal pediatric episodes

\begin{tabular}{|c|c|c|c|}
\hline & $\begin{array}{l}\text { Neonatal episodes } \\
\text { (total } n=113 \text { ) }\end{array}$ & $\begin{array}{l}\text { Non-neonatal pediatric episodes } \\
\text { (total } n=229 \text { ) }\end{array}$ & $P$ value \\
\hline \multicolumn{4}{|l|}{ Clinical features } \\
\hline Severe sepsis & $55(48.7)$ & $71(31.0)$ & 0.002 \\
\hline Septic shock & $39(34.5)$ & $50(21.8)$ & 0.013 \\
\hline Progressive and deteriorated candidiasis $^{a}$ & $27(23.9)$ & $34(14.8)$ & 0.050 \\
\hline Disseminated candidiasis ${ }^{\mathrm{b}}$ & $5(4.4)$ & $9(3.9)$ & 0.828 \\
\hline Breakthrough invasive candidiasis & $10(8.8)$ & $31(13.5)$ & 0.288 \\
\hline \multicolumn{4}{|l|}{ Duration of candidemia and/or persistent invasive fungal infection } \\
\hline Days, median (interquartile range) & $3.0(1.0-6.0)$ & $1.0(1.0-5.0)$ & 0.033 \\
\hline$\leq 2$ days & $48(42.5)$ & $131(57.2)$ & \\
\hline $3-7$ days & $46(40.7)$ & $59(25.8)$ & \\
\hline$\geq 8$ days & 19 (16.8) & $39(17.0)$ & \\
\hline Antifungal regimens for treatment & & & $<0.001$ \\
\hline Fluconazole/Noriconazole & $34(30.1)$ & $97(42.4)$ & \\
\hline Amphotericin B & $50(44.2)$ & $47(20.5)$ & \\
\hline Echinocandin & $20(17.7)$ & $76(33.2)$ & \\
\hline Combination antifungal treatment & $6(5.3)$ & $2(0.9)$ & \\
\hline None & $3(2.7)$ & $7(3.1)$ & \\
\hline Antifungal treatment within $24 \mathrm{~h}$ & $36(31.8)$ & $105(45.9)$ & 0.014 \\
\hline $\begin{array}{l}\text { Duration between onset of invasive candidiasis and initiation } \\
\text { of antifungal agents, mean } \pm S D \text { (days) }\end{array}$ & $2.1 \pm 1.3$ & $1.7 \pm 1.4$ & 0.009 \\
\hline Total treatment duration (days), mean (range) & $17.5(2.0-46.0)$ & $18.9(1.0-68.0)$ & 0.113 \\
\hline Removal of central venous catheter within 3 days of onset & $34 / 108(31.5)$ & $73 / 214(34.1)$ & 0.622 \\
\hline \multicolumn{4}{|l|}{ Treatment outcomes } \\
\hline Responsiveness after initiation of antifungal treatment ${ }^{c}$ & & & 0.157 \\
\hline Within $72 \mathrm{~h}$ & $39(34.5)$ & $96(41.9)$ & \\
\hline 4-7 days & $17(15.0)$ & $47(20.5)$ & \\
\hline More than 7 days & $21(18.6)$ & $41(17.9)$ & \\
\hline Treatment failure & $36(31.0)$ & $45(19.7)$ & 0.015 \\
\hline Modification of antifungal treatment & $44(38.9)$ & $107(46.7)$ & 0.203 \\
\hline Invasive candidiasis attributable mortality & $32(28.3)$ & $40(17.5)$ & 0.024 \\
\hline In-hospital all-cause mortality & $41 / 96(42.7)$ & $47 / 185(25.4)$ & 0.004 \\
\hline
\end{tabular}

All data were expressed as number (percentage \%), unless indicated otherwise

${ }^{a}$ Defined as candidemia episodes with more disseminated candidiasis and/or progressive multi-organ failure even after effective antifungal agents

${ }^{b}$ Indicated positive Candida isolates recovered from more than two sterile sites, in addition to primary bloodstream infection

${ }^{\mathrm{c}}$ Responsiveness was defined according to the consensus criteria of the Mycoses Study Group and European Organization for Research and Treatment of Cancer [29]

greatly in different settings, age groups, or geographic areas $[15,28,30]$. The crude mortality rates are generally lower in younger (pediatric) than older (adult) patients with candidemia regardless of the Candida species $[15,30]$. A recent prospective multicenter surveillance study of candidemia has showed a higher 30-day mortality rate in neonates with candidemia than children $(40 \%$ vs. $28 \%, P=0.02)$ [31]. Our study further demonstrated that neonatal invasive candidiasis has worse responsiveness to antifungal therapy, more prolonged fungemia, more likely to have severe sepsis and septic shock, and higher rates of sepsis attributable mortality and in-hospital mortality.

Several factors can affect the treatment outcomes of candidemia, including underlying chronic comorbidities, microbiological factors, treatment policies and timely administration of antifungal agents, illness severity, and treatment with an infected catheter in situ or removal [3, 14, 32-35]. The underlying illness and predisposing factors for neonatal candidiasis and non-neonatal 


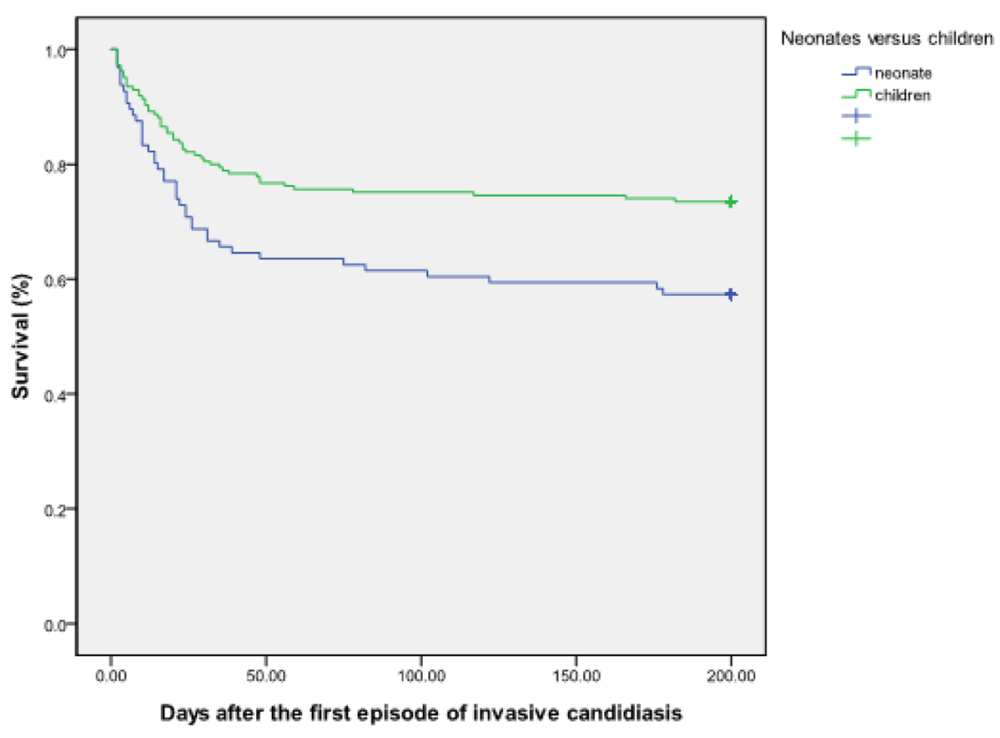

Fig. 2 Survival following the first episode of invasive candidiasis in neonates when compared with children by the Kaplan-Meier method (log-rank test $=0.005)$

pediatric candidiasis are basically different. We found extreme prematurity and related comorbidities, including use of total parenteral nutrition and underlying chronic lung disease to be the major predisposing factors for neonatal candidemia [31]. In non-neonatal pediatric candidiasis, underlying chronic conditions, especially neurological sequelae, hemodialysis, hematological/cancer patients on immunosuppressive treatment and resulting neutropenia accounted for the majority of the susceptible hosts.
Administration of antifungal agents is less frequently delayed in the non-neonatal pediatric setting because the underlying chronic comorbidities would remind clinicians of the possibility of opportunistic infections. Furthermore, we identified incidental cases of congenital candidemia without any of these underlying illnesses or risk factors, which has rarely been reported [36].

Because most of our Candida isolates were sensitive to all commonly prescribed antifungal agents, treatment

Table 4 In vitro susceptibility to various antifungal agents of selected Candida species causing invasive candidiasis in neonatal versus non-neonatal pediatric patients

\begin{tabular}{|c|c|c|c|c|c|c|c|c|c|}
\hline & \multicolumn{3}{|c|}{ All Candida (total $n=295$ ) } & \multicolumn{2}{|l|}{ C. albicans } & \multicolumn{2}{|c|}{ C. parapsilosis } & \multicolumn{2}{|c|}{ Other Candida spp. } \\
\hline & All episodes & Neonates & Children & Neonates & Children & Neonates & Children & Neonates & Children \\
\hline \multicolumn{10}{|l|}{ Fluconazole } \\
\hline Susceptible & $251(85.1)$ & 87 (83.7) & $164(85.9)$ & 47 (97.9) & $73(94.8)$ & $30(100)$ & $59(100)$ & $10(38.5)$ & $32(58.2)$ \\
\hline $\mathrm{S}-\mathrm{DD}$ or $\mathrm{R}$ & $44(17.5)$ & $17(16.3)$ & $27(14.1)$ & $1(2.1)$ & $4(5.2)$ & $0(0)$ & $0(0)$ & $16(61.5)$ & $23(41.8)$ \\
\hline \multicolumn{10}{|l|}{ Voriconazole } \\
\hline Susceptible & $262(88.8)$ & $97(93.3)$ & $165(86.4)$ & $47(97.9)$ & $73(94.8)$ & $30(100)$ & $59(100)$ & $20(76.9)$ & $33(60)$ \\
\hline $\mathrm{S}-\mathrm{DD}$ or $\mathrm{R}$ & $33(11.2)$ & $7(6.7)$ & $26(13.6)$ & $1(2.1)$ & $4(5.2)$ & $0(0)$ & $0(0)$ & $6(23.1)$ & $22(40)$ \\
\hline \multicolumn{10}{|l|}{ Amphotericin B } \\
\hline Susceptible & $292(99.0)$ & $104(100)$ & $188(64.6)$ & $48(100)$ & $77(100)$ & $30(100)$ & $59(100)$ & $26(100)$ & $52(94.5)$ \\
\hline $\mathrm{S}-\mathrm{DD}$ or $\mathrm{R}$ & $3(1.0)$ & $0(0)$ & $3(1.6)$ & $0(0)$ & $0(0)$ & $0(0)$ & $0(0)$ & $0(0)$ & $3(5.5)$ \\
\hline \multicolumn{10}{|l|}{ Micafungin } \\
\hline Susceptible & $291(98.6)$ & $102(98.1)$ & $189(99.0)$ & 48 (100) & 76 (98.7) & 28 (93.3) & $58(98.3)$ & $26(100)$ & $55(100)$ \\
\hline $\mathrm{S}-\mathrm{DD}$ or $\mathrm{R}$ & $4(1.4)$ & $2(1.9)$ & $2(1.0)$ & $0(0)$ & $1(1.3)$ & $2(6.7)$ & $1(1.7)$ & $0(0)$ & $0(0)$ \\
\hline \multicolumn{10}{|l|}{ Caspofungin } \\
\hline Susceptible & $293(99.3)$ & $104(100)$ & $189(99.0)$ & $48(100)$ & 77 (100) & $30(100)$ & $59(100)$ & $26(100)$ & $53(96.4)$ \\
\hline S-DD or R & $2(0.7)$ & $0(0)$ & $2(1.0)$ & $0(0)$ & $0(0)$ & $0(0)$ & $0(0)$ & $0(0)$ & $2(3.6)$ \\
\hline
\end{tabular}

All data were expressed as number (percentage \%), unless indicated otherwise $S$-DD susceptible-dose dependent, $R$ resistant 
Table 5 Risk factors for treatment failure in pediatric invasive candidiasis by univariate and multivariate analysis

\begin{tabular}{|c|c|c|c|c|c|}
\hline \multirow[t]{2}{*}{ Risk factors } & \multicolumn{3}{|l|}{ Univariate analysis } & \multicolumn{2}{|l|}{ Multivariate analysis } \\
\hline & $\begin{array}{l}\text { Treatment success } \\
\text { (total } n=261 \text { ) }\end{array}$ & $\begin{array}{l}\text { Treatment failure } \\
\text { (total } n=81 \text { ) }\end{array}$ & $P$ value & Adjusted OR (95\% Cl) & $P$ value \\
\hline \multicolumn{6}{|l|}{ Neonates vs. children } \\
\hline Neonates & $77(29.5)$ & $36(44.4)$ & 0.015 & $1.96(0.91-4.23)$ & 0.087 \\
\hline Children & $184(70.5)$ & $45(55.6)$ & & 1 (reference) & \\
\hline Initiation of antifungal agents within $24 \mathrm{~h}$ & $100(38.3)$ & $41(50.6)$ & 0.054 & $1.58(0.79-3.16)$ & 0.540 \\
\hline Breakthrough invasive candidiasis & $24(9.2)$ & $17(21.0)$ & 0.010 & $2.99(1.04-8.67)$ & 0.043 \\
\hline Septic shock at onset & $34(13.0)$ & $55(67.9)$ & $<0.001$ & $16.01(7.64-33.56)$ & $<0.001$ \\
\hline Underlying renal failure with/without hemodialysis & $20(7.7)$ & $19(23.5)$ & $<0.001$ & $5.38(1.99-14.57)$ & 0.001 \\
\hline Delayed catheter removal $>3$ days after illness onset & $154(59.0)$ & $71(87.7)$ & $<0.001$ & $6.78(2.48-18.52)$ & $<0.001$ \\
\hline Treatment regimens & & & 0.001 & & \\
\hline Fluconazole & 109 (40.6) & $22(27.2)$ & & 1 (reference) & \\
\hline Amphotericin B & $73(28.0)$ & $24(29.6)$ & & $1.53(0.70-3.33)$ & 0.289 \\
\hline Echinocandin & $73(28.0)$ & $23(28.4)$ & & $1.04(0.47-2.31)$ & 0.933 \\
\hline Combination therapy & $6(2.3)$ & $2(2.5)$ & & $1.76(0.25-12.3)$ & 0.570 \\
\hline No antifungal treatment & $0(0)$ & $10(12.3)$ & & $10.07(1.6-64.7)$ & $<0.001$ \\
\hline Pathogens & & & 0.729 & & \\
\hline Candida albicans & $121(46.4)$ & $34(42.0)$ & & & \\
\hline Candida parapsilosis & $71(27.2)$ & $24(58.0)$ & & & \\
\hline Candida tropicalis & $15(5.7)$ & $7(8.6)$ & & & \\
\hline Candida glabrata & $17(6.5)$ & $4(4.9)$ & & & \\
\hline Other Candida spp. & $36(13.8)$ & $12(14.8)$ & & & \\
\hline Infectious source & & & 0.358 & & \\
\hline Primary bloodstream infection & $169(64.8)$ & $59(72.8)$ & & & \\
\hline Catheter-related bloodstream infection & $59(22.6)$ & $10(12.3)$ & & & \\
\hline Intra-abdominal & $22(8.4)$ & $9(11.1)$ & & & \\
\hline Urological & $4(1.5)$ & $1(1.2)$ & & & \\
\hline Lung & $3(1.1)$ & $1(1.2)$ & & & \\
\hline Meningitis & $4(1.5)$ & $1(1.2)$ & & & \\
\hline
\end{tabular}

failures most likely were caused by the failure of infectious sources control and underlying illness. Our results are similar to those of Grim et al. [37], who concluded that a high mortality rate among patients with candidemia (34\% mortality within 30 days), with underlying cirrhosis and HIV infection, and increased illness severity were the independent risk factors, despite timely receipt of appropriate antifungal therapy. We documented that removal of an infected catheter was an important factor for successful treatment of invasive candidiasis $[38,39]$. Although breakthrough candidemia was not associated with nonsusceptible fluconazole isolates $[8,40]$, it was independently associated with treatment failure and also more prolonged fungemia in our cohort.

This study included all episodes of invasive candidiasis in children [41]. Although some episodes were blood culture-negative intra-abdominal candidiasis and some were Candida spp. positive only in the pleural fluid, all cases of invasive candidiasis were evaluated by the infection specialist and were found to require treatment, unless mortality preceded the diagnosis of invasive fungal infection. Currently no clinical study has assessed the need to treat Candida peritonitis [42, 43], but we excluded cases of probable Candida colonization [44]. Furthermore, the high proportion of mixed-flora peritonitis is one limitation in this study because the pathogenic role of Candida in this polymicrobial form of infection is a matter of debate. However, many experts still consider positive Candida cultures from intraabdominal fluid in patients with peritonitis to be clinically significant even in the presence of concomitant bacterial growth [45]. Antifungal therapy is recommended by the European Society of Clinical Microbiology and Infectious 
Diseases and the Infectious Disease Society of America guidelines on management of complicated intraabdominal abscess or peritonitis that is positive for Candida spp. [46, 47].

Recent studies described an overall mortality of 17.2$46.2 \%$ among children with invasive candidiasis $[15,19-$ $21,30,48-50]$, and mortality attributable to candidemia has been reported to be $12-22 \%$ [15, 30, 48-50]. Some Candida species were associated with worse outcomes. For example, C. tropicalis, C. krusei and C. glabrata related candidemia are associated with higher mortality rates than C. parapsilosis related candidemia in adults [28, 48]. However, our cohort did not include enough patients to support any firm conclusions in the pediatric settings. We found the choice of antifungal therapy did not appear to have a significant impact on treatment result [51-53]. In our cohort, patients who received fluconazole alone had a more favorable outcome than did patients who received other regimens, reflecting a bias toward the administration of fluconazole therapy to patients who were less ill.

The strengths of our study include the systematic identification of specific species of Candida isolates, the systemic collection of clinical data, and the fact that this is the first report that focuses specifically on the difference between neonatal and non-neonatal pediatric settings. However, there were some limitations in this study. First, this study was a retrospective study and conducted in a single center. Therefore, extrapolation of the findings to other institutions must be done cautiously. Second, severity of illness was not documented because the scoring systems were different in the neonatal and non-neonatal period. Third, Candida surveillance cultures were not obtained, and there were no data regarding prior colonization [19]. We failed to document all the risk factors for invasive candidiasis in this study. Furthermore, the policies regarding antifungal therapy may be changed over such an extended study period in our non-interventional study, which should be considered as a description of clinical practice only.

\section{Conclusions}

In conclusion, this study demonstrates some significant differences of invasive candidiasis between neonates and children. Neonatologists and pediatricians must consider age-specific differences when developing treatment and prevention guidelines, or when studies of other age groups are interpreted. Furthermore, because the clinical signs of invasive candidiasis are not specific and early microbiological documentation remains a major challenge, intensive research dedicated to the development of alternative tools for early diagnosis of invasive candidiasis is urgently warranted.

\section{Abbreviations}

BPD: Bronchopulmonary dysplasia; BSI: Bloodstream infection; CDC: Centers for Disease Control and Prevention; Cl: Confidence interval; CRP: C-reactive protein; CVC: Central venous catheter; ESBL: Extended-spectrum $\beta$-lactamase; GBS: Group B streptococcus; GPC: Gram-positive cocci; IQR: Interquartile range; MALDI-TOF: Matrix-assisted laser desorption ionization time-of-flight; NEC: Necrotizing enterocolitis; NICU: Neonatal intensive care unit; NTISS: Neonatal Therapeutic Intervention Scoring System; OR: Odds ratio; PVL: Periventricular leukomalacia; RDS: Respiratory distress syndrome; TPN: Total parenteral nutrition; VAP: Ventilator associated pneumonia

\section{Acknowledgements}

The abstract has been previously published as a conference abstract and accepted as the poster presentation in 2nd Congress of joint European Neonatal Societies (jENS 2017) Session "Neonatal Infectious Diseases/ Immunology" Venice, Italy in October 30- November 4, 2017.

All authors thank financial support from the Chang Gung Medical Research Program Foundation (grants CMRPG3E1491). We thank Professor Jang-Jih Lu (Department of Laboratory Medicine, Department of Medical Biotechnology and Laboratory Science Chang Gung Memorial Hospital at Linkou, Taoyuan, Taiwan) for providing the technical support of all Candida isolates re-identification and antifungal susceptibility testing.

\section{Funding}

Chang Gung Medical Research Program Foundation (grants CMRPG3E1491). The role of the funding body is to support the isolation and identification of all Candida spp. isolates in blood and sterile site cultures, and to perform the antifungal susceptibility testing.

\section{Availability of data and materials}

The datasets used/or analyzed during the current study available from the corresponding author on reasonable request.

\section{Authors' contributions}

Conceptualization: JFH, MYL, CWL, SMC. Data collection and verification: JFH, MYL, SMC, HRH, IHW, ITL, CMC, RHF. Formal analysis: JFH, CWL, ITL. Funding acquisition: JFH, MHT. Investigation: JFH, MYL, SMC, HRH, IHW, ITL, CMC, RHF. Methodology: JFH, MYL, SMC, HRH, IHW, ITL, CMC, RHF. Supervision: MHT. Writing - original draft: JFH, MHT. Writing - review \& editing: MHT. All authors have read and approve of the final version of the manuscript.

\section{Ethics approval and consent to participate}

This study was approved by the institutional review board of Chang Gung Memorial Hospital, with a waiver of informed consent because all patient records and information were anonymized and de-identified prior to analysis.

\section{Competing interests}

The authors declare that they have no competing interests.

\section{Publisher's Note}

Springer Nature remains neutral with regard to jurisdictional claims in published maps and institutional affiliations.

\section{Author details}

'Division of Pediatric Neonatology, Department of Pediatrics, Chang Gung Memorial Hospital, Taoyuan, Taiwan. ${ }^{2}$ Division of Neonatology and Pediatric Hematology/Oncology, Department of Pediatrics, Chang Gung Memorial Hospital, No.707, Gongye Rd., Sansheng, Mailiao Township, Yunlin, Taiwan, Republic of China. ${ }^{3}$ College of Medicine, Chang Gung University, Taoyuan, Taiwan. ${ }^{4}$ Department of Nursing, Division of Basic Medical Sciences and Research Center for Industry of Human Ecology, Chang Gung University of Science and Technology, Chiayi, Taiwan. ${ }^{5}$ Department of Medical Research, Taichung Veterans General Hospital, Taichung, Taiwan. 


\section{Received: 15 September 2017 Accepted: 17 April 2018 Published online: 24 April 2018}

\section{References}

1. Tan BH, Chakrabarti A, Li RY, Patel AK, Watcharananan SP, Liu Z, et al. Incidence and species distribution of candidaemia in Asia: a laboratorybased surveillance study. Clin Microbiol Infect. 2015;21:946-53.

2. Reichert F, Piening B, Geffers C, Gastmeier P, Bührer C, Schwab F. Pathogenspecific clustering of nosocomial blood stream infections in very preterm infants. Pediatrics. 2016;137.

3. Fox ML, Barba P, Heras I, López-Parra M, González-Vicent M, de la Cámara R, et al. A registry-based study of non-aspergillus mould infections in recipients of allogeneic haematopoietic cell transplantation. Clan Microbiol Infect. 2015;21:e1-3.

4. Tsai MH, Wang SH, Hsu JF, Lin LC, Chu SM, Huang HR, et al. Clinical and molecular characteristics of bloodstream infections caused by Candida albicans in children from 2003 to 2011. Clin Microbiol Infect. 2015;21:1018.e1.

5. Theocharidou E, Agarwal B, Jeffrey G, Jalan R, Harrison D, Burroughs AK, et al. Early invasive fungal infections and colonization in patients with cirrhosis admitted to the intensive care unit. Clin Microbiol Infect. 2015;22: 189.e1.

6. Lortholary O, Renaudat C, Sitbon K, Madec Y, Denoeud-Ndam L, Wolff M, et al. Worrisome trends in incidence and mortality of candidemia in intensive care units (Paris area, 2002-2010). Intensive Care Med. 2014;40: 1303-12.

7. Hesstvedt L, Gaustad P, Andersen CT, Haarr E, Hannula R, Haukland HH, et al. Twenty-two years of candidaemia surveillance: results from a Norwegian national study. Clin Microbiol Infect. 2015;21:938-45.

8. Cuervo G, Garcia-Vidal C, Nucci M, Puchades F, Fernandez-Ruiz M, Obed M et al. Breakthrough candidaemia in the era of broad-spectrum antifungal therapies. Clin Microbiol Infect. 2015;22:181-8.

9. Oeser C, Vergnano S, Naidoo R, Anthony M, Chang J, Chow P, et al. Neonatal invasive fungal infection in England 2004-2010. Clin Microbiol Infect. 2014;20:936-41.

10. Klingspor L, Tortorano AM, Peman J, Willinger B, Hamal P, Sendid B, et al. Invasive candida infections in surgical patients in intensive care units: a prospective, multicentre survey initiated by the European Confederation of Medical Mycology (ECMM) (2006-2008). Clin Microbiol Infect. 2015;21:87-e1.

11. Colombo AL, Guimarães T, Sukienik T, Pasqualotto AC, Andreotti R, QueirozTelles F, et al. Prognostic factors and historical trends in the epidemiology of candidemia in critically ill patients: an analysis of five multicenter studies sequentially conducted over a 9-year period. Intensive Care Med. 2014;40: 1489-98.

12. Cuervo G, Puig-Asensio M, Garcia-Vidal C, Fernandez-Ruiz M, Peman J, Nucci $M$, et al. A simple prediction score for estimating the risk of candidaemia caused by fluconazole non-susceptible strains. Clin Microbiol Infect. 2015;21: 684.e1.

13. Asmundsdottir LR, Erlendsdottir $H$, Gottfredsson M. Nationwide study of candidemia, antifungal use, and antifungal drug resistance in Iceland, 2000 to 2011. J Clin Microbiol. 2013;51:841-8.

14. Puig-Asensio M, Padilla B, Garnacho-Montero J, Zaragoza O, Aguado JM, Zaragoza R, et al. Epidemiology and predictive factors for early and late mortality in Candida bloodstream infections: a population-based surveillance in Spain. Clin Microbiol Infect. 2014;20:0245-54.

15. Blyth CC, Chen SC, Slavin MA, Serena C, Nguyen Q, Marriott D, et al. Not just little adults: candidemia epidemiology, molecular characterization, and antifungal susceptibility in neonatal and pediatric patients. Pediatrics. 2009; 123:1360-8.

16. Taj-Aldeen SJ, Kolecka A, Boesten R, Alolaqi A, Almaslamani M, Chandra P, et al. Epidemiology of candidemia in Qatar, the Middle East: performance of MALDI-TOF MS for the identification of Candida species, species distribution, outcome, and susceptibility pattern. Infection. 2014;42:393-404.

17. Hsu JF, Chu SM, Huang YC, Lien R, Huang HR, Lee CW, et al. Predictors of clinical and microbiological treatment failure in neonatal bloodstream infections. Clin Microbiol Infect. 2015;21:482.e9.

18. Abelson JA, Moore T, Bruckner D, Deville J, Nielsen K. Frequency of fungemia in hospitalized pediatric inpatients over 11 years at a tertiary care institution. Pediatrics. 2005;116:61-7.

19. Vogiatzi L, llia S, Sideri G, Vagelakoudi E, Vassilopoulou M, Sdougka M, et al. Invasive candidiasis in pediatric intensive care in Greece: a nationwide study. Intensive Care Med. 2013;39:2188-95.
20. Dotis J, Prasad PA, Zaoutis T, Roilides E. Epidemiology, risk factors and outcome of Candida parapsilosis bloodstream infection in children. Pediatr Infect Dis J. 2012;31:557-60.

21. Tragiannidis A, Fegeler W, Rellensmann G, Debus V, Müller V, Hoernig-Franz I, et al. Candidaemia in a European Paediatric university hospital: a 10-year observational study. Clin Microbiol Infect. 2012;18:E27-30.

22. Orasch C, Marchetti O, Garbino J, Schrenzel J, Zimmerli S, Mühlethaler K, et al. Candida species distribution and antifungal susceptibility testing according to European committee on antimicrobial susceptibility testing and new vs. old clinical and laboratory standards institute clinical breakpoints: a 6-year prospective candidaemia survey from the fungal infection network of Switzerland. Clin Microbiol Infect. 2014;20:698-705.

23. Jung DS, Farmakiotis D, Jiang Y, Tarrand JJ, Kontoyiannis DP. Uncommon candida species fungemia among cancer patients, Houston, Texas, USA. Emerg Infect Dis. 2015;21:1942-50.

24. Steinbach WJ, Foilides E, Berman D, Hoffman JA, Groll AH, Bin-Hussain I, et al. Results from a prospective, international, epidemiologic study of invasive candidiasis in children and neonates. Pediatr Infect Dis J. 2012;31: 1252-7.

25. Nguyen MH, Wissel MC, Shields RK, Salomoni MA, Binghua $H$, Press EG, et al. Performance of Candida real-time polymerase chain reaction, $\beta$-D-glucan assay, and blood cultures in the diagnosis of invasive candidiasis. Clin Infect Dis. 2012;54:1240-8.

26. Muñoz P, Vena A, Valerio M, Álvarez-Uria A, Guinea J, Escribano P, et al. Risk factors for late recurrent candidaemia. A retrospective matched case-control study. Clin Microbiol Infect. 2016;22:277.e11.

27. Lerolle N, Raffoux E, Socie G, Touratier S, Sauvageon H, Porcher R, et al. Breakthrough invasive fungal disease in patients receiving posaconazole primary prophylaxis: a 4-year study. Clin Microbiol Infect. 2014;20:0952-9.

28. Horn DL, Neofytos D, Anaissie EJ, Fishman JA, Steinbach WJ, Olyaei AJ, et al. Epidemiology and outcomes of candidemia in 2019 patients: data from the prospective antifungal therapy alliance registry. Clin Infect Dis. 2009;48: 1695-703.

29. Segal BH, Herbrecht R, Stevens DA, Ostrosky-Zeichner L, Sobel J, Viscoli C, et al. Defining responses to therapy and study outcomes in clinical trials of invasive fungal diseases: mycoses study group and European Organization for Research and Treatment of Cancer consensus criteria. Clin Infect Dis. 2008:47:674-83.

30. Zaoutis TE, Argon J, Chu J, Berlin JA, Walsh TJ, Feudtner C. The epidemiology and attributable outcomes of candidemia in adults and children hospitalized in the United States: a propensity analysis. Clin Infect Dis. 2005;41:1232-9.

31. Santolaya ME, Alvarado T, Queiroz-Telles F, Colombo AL, Zurita J, Tiraboschi IN, et al. Active surveillance of candidemia in children from Latin America: a key requirement for improving disease outcome. Pediatr Infect Dis J. 2014; 33:e40-4.

32. Gamaletsou MN, Walsh TJ, Zaoutis T, Pagoni M, Kotsopoulou M, Voulgarelis $\mathrm{M}$, et al. A prospective, cohort, multicentre study of candidaemia in hospitalized adult patients with haematological malignancies. Clin Microbiol Infect. 2014;20:050-7.

33. Farmakiotis D, Kyvernitakis A, Tarrand JJ, Kontoyiannis DP. Early initiation of appropriate treatment is associated with increased survival in cancer patients with Candida glabrata fungaemia: a potential benefit from infectious disease consultation. Clin Microbiol Infect. 2015;21:79-86.

34. Beyda ND, John J, Kilic A, Alam MJ, Lasco TM, Garey KW. FKS mutant Candida glabrata: risk factors and outcomes in patients with candidemia. Clin Infect Dis. 2014;59:819-25.

35. Kollef M, Micek S, Hampton N, Doherty JA, Kumar A. Septic shock attributed to Candida infection: importance of empiric therapy and source control. Clin Infect Dis. 2012;54:1739-46.

36. Carmo KB, Evans N, Isaacs D. Congenital candidiasis presenting as septic shock without rash. Arch Dis Child. 2007;92:627-8.

37. Grim SA, Berger K, Teng C, Gupta S, Layden JE, Janda WM, et al. Timing of susceptibility-based antifungal drug administration in patients with Candida bloodstream infection: correlation with outcomes. J Antimicrob Chemother. 2012;67:707-14.

38. Puig-Asensio M, Pemán J, Zaragoza R, Garnacho-Montero J, Martin-Mazuelos E, Cuenca-Estrella M, et al. Impact of therapeutic strategies on the prognosis of candidemia in the ICU. Crit Care Med. 2014;42:1423-32.

39. Garnacho-Montero J, Díaz-Martín A, Garcia-Cabrera E, Ruiz Pérez de Pipaón M, Hernández-Caballero C, Lepe-Jiménez JA. Impact on hospital mortality of 
catheter removal and adequate antifungal therapy in Candida spp. bloodstream infections. J Antimicrob Chemother. 2013;68:206-13.

40. Pasqualotto AC, Nedel WL, Machado TS, Severo LC. Risk factors and outcome for nosocomial breakthrough candidaemia. J Inf Secur. 2006;52: 216-22.

41. Pilmis B, Puel A, Lortholary O, Lantemier F. New clinical phenotypes of fungal infections in special hosts. Clin Microbiol Infect. 2016;22:681-7.

42. Zappella N, Desmard M, Chochillon C, Ribeiro-Parenti L, Houze S, Marmuse $J \mathrm{P}$, et al. Positive peritoneal fluid fungal cultures in postoperative peritonitis after bariatric surgery. Clin Microbiol Infect. 2015;21:853.e1.

43. Tissot F, Lamoth F, Hauser PM, Orasch C, Flückiger U, Siegemund M, et al. $\beta$-Glucan antigenemia anticipates diagnosis of blood culture-negative intraabdominal candidiasis. Am J Respir Crit Care Med. 2013;188:1100-9.

44. Terraneo S, Ferrer M, Martin-Loeches I, Esperatti M, Di Pasquale M, Giunta V, et al. Impact of Candida spp. isolation in the respiratory tract in patients with intensive care unit-acquired pneumonia. Clin Microbiol Infect. 2016;22:94.e1.

45. Montravers P, Mira JP, Gangneux JP, Leroy O, Lortholary O. A multicentre study of antifungal strategies and outcome of Candida spp. peritonitis in intensive-care units. Clin Microbiol Infect. 2011;17:1061-7.

46. Pappas PG, Kauffman CA, Andes DR, Clancy CJ, Marr KA, Ostrosky-Zeichner $L$, et al. Clinical practice guideline for the management of candidiasis: 2016 update by the infectious disease Society of America. Clin Infect Dis. 2015;62:e1-50

47. Cuenca-Estrella M, Verweij PE, Arendrup MC, Arikan-Akdagli S, Bille J, Donnelly JP, et al. ESCMID guideline for the diagnosis and management of Candida disease 2012: diagnostic procedures. Clin Microbiol Infect. 2012; 18(Suppl 7):9-18.

48. Zaoutis T. Candidemia in children. Curr Med Res Opin. 2010;26:1761-8.

49. Sutcu M, Salman N, Akturk H, Dalgıc N, Turel O, Kuzdan C, et al. Epidemiologic and microbiologic evaluation of nosocomial infection associated with Candida spp in children: a multicenter study from Istanbul, Turkey. Am J Infect Control. 2016:44:1139-43.

50. Tortorano AM, Dho G, Prigitano A, Breda G, Grancini A, Emmi V, et al. Invasive fungal infections in the intensive care unit: a multicentre, prospective, observational study in Italy (2006-2008). Mycoses. 2012;55:73-9.

51. Pappas PG, Rex JH, Lee J, Hamill RJ, Larsen RA, Powderly W, et al. A prospective observational study of candidemia: epidemiology, therapy, and influences on mortality in hospitalized adult and pediatric patients. Clin Infect Dis. 2003:37:634-43.

52. Puig-Asensio M, Fernández-Ruiz M, Aguado JM, Merino P, Lora-Pablos $D$, Guinea J, et al. Propensity score analysis of the role of initial antifungal therapy in the outcome of Candida glabrata bloodstream infections. Antimicrob Agents Chemother. 2016;60:3291-300.

53. Fernández-Ruiz M, Aguado JM, Almirante B, Lora-Pablos D, Padilla B, Puig-Asensio M, et al. Initial use of echinocandins does not negatively influence outcome in Candida parapsilosis bloodstream infection: a propensity score analysis. Clin Infect Dis. 2014;58:1413-21.

\section{Ready to submit your research? Choose BMC and benefit from:}

- fast, convenient online submission

- thorough peer review by experienced researchers in your field

- rapid publication on acceptance

- support for research data, including large and complex data types

- gold Open Access which fosters wider collaboration and increased citations - maximum visibility for your research: over $100 \mathrm{M}$ website views per year

At BMC, research is always in progress.

Learn more biomedcentral.com/submissions 